\title{
THE EXISTENCE OF CERTAIN TYPES OF MANIFOLDS
}

BY

\section{L. CURTIS AND R. L. WILDER( $\left.{ }^{1}\right)$}

Introduction. The first part of the paper gives a class of polyhedral 3manifolds in the 4-sphere $S^{4}$ and with homology groups $H_{1}(M)=H_{2}(M)=0$, but with $\pi_{1}(M) \neq 0$. Thus some "Poincare" spaces are imbeddable in $S^{4}$. Also, necessary and sufficient conditions are given for a group to be $H_{1}(M)$ for some 3-manifold $M$ in $S^{4}$. In Part Two a negative answer is found for the following question: Since the metric cases of 2 -gcms reduce to the classical types [12, IX], and the 3 -gcms generally do not, can one state that a spherelike 3 -gcm in $S^{4}$ must be a classical manifold? We construct 3 -gcms in $S^{4}$ which have the same homology and homotopy character as the 3-sphere (globally and locally), but which are not locally euclidean. In part three of the paper a negative answer is found for a question proposed by Griffiths [6] by showing the existence of a 3-dimensional "homotopy manifold" which is not locally euclidean. This is a space previously obtained by R. H. Bing by identifying the points on a certain set of tame arcs in $S^{3}$.

Part I. Let $P$ be a connected 2-polyhedron which is contained in $S^{4}$ as a subcomplex of some simplicial decomposition $\Sigma$ of $S^{4}$. Before anything else is done we make a barycentric subdivision of $\Sigma$. This is to make sure that the resulting decomposition of $P$ will be complete in the sense that if all of the vertices of a simplex of $S^{4}$ lie in $P$, then the simplex itself is in $P$.

Now every simplex of $\operatorname{St}(P)-P$ is the join of a simplex of $P$ with a simplex in $S^{4}-\mathrm{St}(P)$. Hence each $x \in(\operatorname{St}(P)-P)$ may be assigned a number $t(x)$ between 0 and 1 , its "distance from $P$ ". Newman [8] has shown that the set $M=[x \mid t(x)=1 / 2]$ is a manifold. The set $M$ is clearly 3 -dimensional, polyhedral and orientable. If we let $X=[x \mid 0 \leqq t(x)<1 / 2]$, then $\bar{X}, X$ and $P$ all have the same homotopy type. The homology groups of $M$ and $P$ are related in the following manner.

Proposition I.1. If $H_{2}(P, \mathfrak{p})=0$, where $\mathfrak{p}$ is the additive group of reals modulo 1 , then $H_{1}(M, Z)=H_{1}(P, Z)$, where $Z$ is the additive group of integers.

Proof. Let $Y=S^{4}-\bar{X}$. By the Mayer-Vietoris theorem,

$$
H_{1}(M, Z) \cong H_{1}(\bar{X}, Z)+H_{1}(\bar{Y}, Z) .
$$

Presented to Society, December 28, 1955; received by the editors January 23, 1957, and, in revised form, June 6, 1957.

(1) This paper is a result of joint work by the authors at the Set Theoretic Topology Institute at Madison, Wisconsin, in 1955. 
If $H_{2}(P, \mathfrak{p})=0$, then $H_{2}(X, \mathfrak{p})=0$, and $H_{1}(\bar{Y}, Z)=0$ since $H_{2}(X, \mathfrak{p})$ and $H_{1}(\bar{Y}, Z)$ are dually paired. Hence (1) gives

$$
H_{1}(M, Z) \cong H_{1}(\bar{X}, Z) \cong H_{1}(P, Z) .
$$

In the remainder of the paper, $H_{1}(M)$ will always be taken to mean $H_{1}(M, Z)$.

The natural contraction of $\bar{X}$ onto $P$ induces a map $\phi: M \rightarrow P$, and this induces a homomorphism on the fundamental group of $M$.

Proposition I.2. The homomorphism $\phi_{\sharp}: \pi_{1}(M) \rightarrow \pi_{1}(P)$ induced by $\phi$ is onto.

Proof. We apply the type of argument given in [8]. If $s: I \rightarrow P$ is a closed edge path in $P$, we choose for each edge $\sigma^{\prime}$, an edge $\tilde{\sigma}^{\prime}$ in $M$ such that $\phi\left(\tilde{\sigma}^{\prime}\right)$ $=\sigma^{\prime}$. Next the edges are to be connected so that we get an edge path $\tilde{s}$ in $M$ such that $\phi(\tilde{s})=s$. This will prove the lemma, and it can clearly be done if we show that for each vertex $v$ in $P, \phi^{-1}(v)$ is connected.

Considering the decomposition of $S^{4}$ utilizing the vertices of $M$ as well as all vertices in the previous decomposition, we have that $\phi^{-1}(v)=\beta(\operatorname{St}(v))$ $-\mathrm{St}(P), \beta(\mathrm{St}(v))$ denoting the boundary of the star of $v$. We show that $P$ does not separate $\beta(\operatorname{St}(v))$ since $\beta(\operatorname{St}(v))$ is a 3 -sphere and $\beta(\operatorname{St}(v)) \cap P$ is at most 1-dimensional. If $\sigma^{2}$ were a 2 -simplex in $\beta(\operatorname{St}(v)) \cap P$ then the join $\sigma^{2} \bigvee v$ is a 3-simplex whose vertices lie in $P$. Since $P$ is complete $\sigma^{2} \bigvee v$ is in $P$, contradicting the 2-dimensionality of $P$. Since $P$ does not separate $\beta(\operatorname{St}(v)$ ), neither does $\operatorname{St}(P)$ and $\phi^{-1}(v)=\beta(\operatorname{St}(v))-\operatorname{St}(P)$ is connected. This proves the proposition.

We now construct some 2-polyhedra in $S^{4}$ to which we can apply the two propositions of this part.

(1) $A$ disk in $S^{4}$ whose boundary is $n$ times a simple closed curve.

This is the obvious generalization of a cross-cap. Divide the half-circular disk shown into $2 n$ equal sectors.

It is clear that thecurved boundaries of sectors $0 p_{2 k-1} p_{2 k} p_{2 k+1}(k=1, \cdots, n)$ can be identified in $E^{3}$ without any other identifications, that is, without any self intersections except at the curved boundary, so that each are $p_{2 k-1} p_{2 k} p_{2 k+1}$ becomes a simple closed curve, and a preassigned orientation is preserved along $p_{1} p_{2} \cdots p_{2 n+1}$. Now consider $E^{3}$ in $E^{4}$ and choose a point $q \in E^{4}-E^{3}$. We add to the polyhedron the join of $q$ with the simple closed curve $p_{1} 0 p_{2 n+1}$. The resulting polyhedron is of the desired type.

Application. Given any finitely-generated abelian group $G$ we take a basis $g_{1}, g_{2}, \cdots, g_{n}$. Then the relations are all of the form $k_{i} g_{i}=0$ (with the convention that $k_{i}=\infty$ means that no multiple of $g_{i}$ is the identity). We use coordinates $x, y, z, w$ in $E^{4}$. In the $x y$ plane we take $n$ simple closed curves with a single common point. Call these $c_{1}, \cdots, c_{n}$. For each $i$ we attach a disk in $E^{4}$ whose boundary is $k_{i}$ times $c_{i}$. For $k_{i}=\infty$ no disk is attached. The polyhedron $P$ so constructed is connected, 2-dimensional, and $H_{1}(P)=G$. 


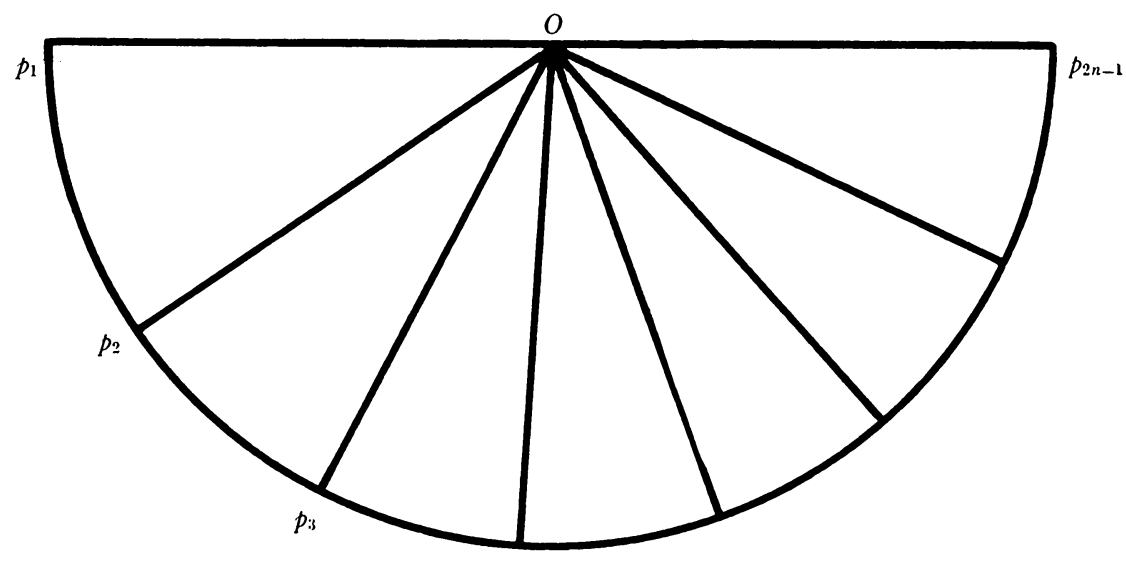

Figure 1

Theorem I.1.( $\left.{ }^{2}\right)$ Let $G$ be a finitely-generated abelian group. Then $G$ is isomorphic with $H_{1}(M, Z)$ for some orientable polyhedral 3-manifold $M$ in $S^{4}$ if and only if the torsion subgroup of $G$ is the direct sum of two isomorphic groups.

Proof. Suppose $M$ is such a manifold in $S^{4}$. Let $X$ and $Y$ be the complementary domains. Then by the Mayer-Vietoris theorem

$$
H_{1}(M, Z) \approx H_{1}(\bar{X}, Z)+H_{1}(\bar{Y}, Z) .
$$

By the Alexander duality theorem the torsion subgroups are such that

$$
T_{1}(\bar{X}, Z) \approx T_{1}(Y, Z) \approx T_{1}(\bar{Y}, Z)
$$

so that

$$
T_{1}(M, Z) \approx T_{1}(\bar{X}, Z)+T_{1}(\bar{X}, Z) .
$$

Conversely, suppose $G=F+T+T$ where $T+T$ is the torsion subgroup of $G$. We use the technique described above to construct a polyhedron $P$ having $H_{1}(P, Z)=F+T$. Then for the corresponding manifold $M$ constructed as in the introductory paragraphs of Part I, we have

$$
H_{1}(M, Z) \approx H_{1}(P, Z)+H_{1}(Y, Z) \text {. }
$$

Furthermore, $H_{1}(Y, Z)$ is a torsion group as we see by applying the Alexander duality to $\bar{X}$ and $Y$ using a field of characteristic zero as coefficients. Hence $H_{1}(Y, Z)=T_{1}(Y, Z)$ and, again using the Alexander duality,

$$
T_{1}(Y, Z) \approx T_{1}(\bar{X}, Z) \approx T_{1}(P, Z) \approx T .
$$

Hence

(2) We are indebted to the referee for pointing out this theorem which replaces an incorrect version. 


$$
H_{1}(M, Z) \approx F+T+T=G .
$$

(2) A polyhedron $P$ in $S^{4}$ having an arbitrary (but finitely-presentable) group as $\pi_{1}(P)$.

Suppose the given group $\pi$ has the presentation $\left(g_{1}, \cdots, g_{k} \mid r_{1}, \cdots, r_{q}\right)$. We take simple closed curves $c_{1}, \cdots, c_{k}$ in the $x y$ plane having the origin as the only intersection. Each relator $r_{j}$ is a word in the $g_{i}$ 's and we let $a_{j}$ denote the number of letters in the word $r_{j}$. For each $r_{j}$ we take $a_{j}$ sectors $s_{1}, \cdots, s_{a_{j}}$ and identify the curved boundary of each $s_{t}$ with the simple closed curve corresponding to the $t$ th letter in $r_{j}$. Next, if the $t$ th and the $(t+1)$ st letter are the same we identify the last radial edge of $s_{t}$ with the first radial edge of $s_{t+1}$. The points of the sectors at the centers of the corresponding circles are identified. This can all be accomplished in $E^{3}$ without any anwanted intersections. Each time the $t$ th and the $(t+1)$ st letters are different we are left with an "eye", and the same is true at the end of each word. Each eye is to be closed by taking the join of it with a point outside of $E^{3}$ in $E^{4}$. These joins can all be made disjoint (except at the common vertex of the $s_{t}$ 's and the origin). By the construction $\pi_{1}(P)=\pi$.

THEOREM I.2. There exist 3-manifolds $M$ in $E^{4}$ with $H_{1}(M)=0$ but with $\pi_{1}(M) \neq 0$.

Proof. Let $\pi$ be a finitely-presentable perfect group. We construct $P$ and $M$ as above with $\pi_{1}(P)=\pi$. Since $\pi_{1}(M)$ maps onto $\pi_{1}(P)$, it follows that $\pi_{1}(M) \neq 0$. However, since $\pi$ is perfect $H_{1}(P)=0$. Hence to complete the proof, we must simply exhibit a perfect group which will make the corresponding $P$ such that $H_{2}(P, \mathfrak{p})=0$; then by Proposition $I .1, H_{1}(M)=0$. We take generators $a, b$ and relations $a^{-3}(a b)^{2}=1, b^{-5}(a b)^{2}=1$, and a routine calculation gives $H_{2}(P, Z)=0$. Now $H_{2}(P, \mathfrak{p})=H_{2}(P, Z) \otimes \mathfrak{p}+$ Tor $\left(H_{1}(P, Z), \mathfrak{p}\right)$ by the universal coefficient theorem. Since $H_{1}(P, Z)=0$, we get the desired result.

Part II. It is known [13] that if $M$ is an orientable $n$-gcm and $f(M)=M^{\prime}$ is an $(n-1)$-monotone mapping of $M$ onto a Hausdorff space $M^{\prime}$, then $M^{\prime}$ is an orientable $n$-gcm of the same homology type as $M$. Consequently one may hope to obtain $n$-gcms having curious properties by the simple device of identification of points in acyclic subsets of ordinary manifolds.

One such class of identifications is that wherein one identifies all points on a "wild" arc in ordinary 3-space.

Let $C$ be an arc in $E^{3}$. If $C$ is shrunk to a point the resulting space may not be $E^{3}$. However, if $C$ is put in $E^{4}$ by putting $E^{3}$ in $E^{4}$ and then $C$ is shrunk to a point, the resulting space is $E^{4}$. To show this we use the following lemma due to Klee [7, Lemma 3.3].

Lemмa. If the linear space $L$ is represented as a sum $L=L_{1}+L_{2}, C$ is a closed subset of $L_{2}$, and $h$ is a homeomorphism of $C$ into $L_{1}$, then there exists an 
isotopy of $L$ onto itself, the initial stage of the isotopy being the identity and the final stage being $h$ on $C$.

Let $E^{4}=E^{3}+E^{1}$ and $h$ be a homeomorphism of $C$ (in $E^{3}$ ) onto the unit interval in $E^{1}$. Let $f: E^{4} \rightarrow E^{4}$ be the final stage of the isotopy furnished by the lemma, and let $g_{1}$ and $g_{2}$ be the quotient maps of $E^{4}$ obtained by shrinking $C$ and $h(C)$ to points. Now $g_{2}\left(E^{4}\right)$ is homeomorphic with $E^{4}$ since $h(C)$ is a linear interval. The map $g_{2} f g_{1}^{-1}$ is a homeomorphism of $g_{1}\left(E^{4}\right)$ to $g_{2}\left(E^{4}\right)$ and our assertion is proved. This means that an arc which is wild in $E^{3}$ will have to be tame in $E^{4}$.

In this part of the paper we use this result to obtain some non-euclidean generalized manifolds in the 4-sphere. In particular we shrink some of the Artin-Fox wild arcs [1].

(1) $A 3-\mathrm{gcm} M$ in $S^{4}$ which has the homology and homotopy of $S^{3}$, is locally euclidean except at a point $p$, and $M-p$ is not simply connected.

This is obtained by shrinking the arc of example 1.1 of [1]. The fact that $M$ has the homotopy type of $S^{3}$ follows from a theorem of J. H. C. Whitehead $\left[10\right.$, p. 1126]. The map $g_{2} f g_{1}^{-1}$ imbeds $M$ in $S^{4}$, and the other conclusions are clear from the properties of the arc used.

It should be noted that not only does the resulting $3-\mathrm{gcm} M$ have the properties stated, but if $U$ is any neighborhood of $p$ in $M$, then $U-p$ contains a singular 1-sphere that is not contractible in $M-p$; i.e., $M-p$ is not $1-L C$ at $p[4$, p. 614].

(2) Example 1.2 of [1] gives an $M$ which is homeomorphic with $S^{3}$, because the complement of the arc is an open 3-cell. This shows, incidentally, that an arc may be wild, yet its image in the quotient space obtained by identification of its points may not be wild.

(3) $A 3-\mathrm{gcm} M$ in $S^{4}$ which has the homotopy type of $S^{3}$ and contains a point $p$ such that $M-p$ is locally euclidean and simply connected, but such that $M-p$ is not $1-L C$ at $p$.

This is obtained by using example 1.3 of [1].

(4) If example 1.4 of [1] is used, the result is $S^{3}$ just as in the case of example 1.2. To see this we must show that the complement of the arc $H^{\natural}$ is an open 3-cell. Let $A_{1}=I I^{\sharp}, A_{2}=H^{b}$ and note that $A_{1} \cap A_{2}=q$. Let $T_{i}(i=1,2)$ be a tubular neighborhood of $A_{i}-q$ (closely approximating this set), such that $\bar{T}_{1} \cap \bar{T}_{2}=q$. There is a homeomorphism $h_{i}$ of $\bar{T}_{i}$ onto the 3 -cell $E$ $\left(x^{2}+y^{2}+z^{2} \leqq 1\right)$ which carries $q$ into $(1,0,0)$ and $A_{i}$ onto the interval $I$ $(0 \leqq x \leqq 1, y=0=z)$. Also, there exists a mapping $g(E)=E$ which leaves points on the boundary of $E$ fixed, is a homeomorphism on $E-I$, and carries $I$ into $(1,0,0)$ (compare with $[1,984]$ ).

The mapping $f$ which is (1) the identity on $S^{3}-\left(T_{1} \cup T_{2}\right),(2) h_{i}^{-1} g h_{i}$ on $T_{i}, i=1,2$, is a homeomorphism on $S^{3}-H^{\natural}$ and carries $H^{\natural}$ into $q$. Hence $S^{3}-H^{\natural}$ is an open 3 -cell.

Part III. The examples of non-euclidean generalized manifolds in Part II 
can all be distinguished from classical manifolds by some local homotopy properties. In this part we show that a certain example used by Bing [3] is a non-euclidean 3-dimensional manifold which has nicer local homotopy properties $\left({ }^{3}\right)$. In particular, it is a homotopy manifold as defined by Griffiths [6].

Let $f: E^{3} \rightarrow X$ be the mapping of $E^{3}$ induced by the decomposition of $E^{3}$ into points and tame arcs as defined by Bing [3]. As Bing shows, $X$ is not homeomorphic with $E^{3}$. Let

$$
C=\left[x \mid f^{-1}(x) \text { is an arc }\right] .
$$

Then $C$ is a Cantor set, and consists of those points of $X$ at which $X$ is not locally euclidean. Let $x \in C$ and suppose $x$ has a neighborhood $U$ homeomorphic with $E^{3}$. Referring to the manner of construction, we see that there exists in $U$ a neighborhood of $x$ whose boundary is a homeomorph of one of the "figure eight" surfaces closing down on $f^{-1}(x)$, say $A_{i j} \ldots n$. Let $A_{i j \ldots n}$ be the " $A$ " of Bing's construction, with $f^{-1}(U)$ taking the place of his decomposition space; then the argument given in $[3, \S 8]$ applies to show that $U$ cannot be homeomorphic with $E^{3}$.

Lemma 3.1. $\pi_{k}(X)=0$ for all $k$, and $X$ is $L C^{\infty}$.

This follows from a theorem $\left({ }^{4}\right)$ of Smale [9] which implies that all of the homotopy groups of $X$ must vanish, and that $X$ is $L C^{\infty}$.

To show that $X$ is a homotopy manifold, we need principally to show that if $x \in X$ and $U$ is a neighborhood of $x$, then there exist neighborhoods $V$ and $W$ of $x$ such that $W \subset V \subset U$, and if $j:(W-x) \rightarrow(V-x)$ is the inclusion map, then the induced homomorphism $j_{*}$ is such that each image $j_{*}\left(\pi_{k}(W-x)\right)$ is isomorphic with $\pi_{k}\left(S^{2}\right)$. Since this is certainly the case if $x \in C$, we confine our attention to the case $x \in C$.

Let $\gamma=f^{-1}(x)$, and let $\left\{T_{i}\right\}$ denote the sequence of figure eight neighborhoods that close down on $\gamma([3])$. Let $\left\{E_{i}\right\}$ be a sequence of 3-cell neighborhoods of $\gamma$ such that (1) $T_{i} \supset E_{i} \supset T_{i+1}$, (2) $E_{i}-\gamma$ is homotopy equivalent to $S^{2}$ (recall that $\gamma$ is a tame arc of $E^{3}$ ). We observe that each $D_{i}=f\left(T_{i}\right)$ is a neighborhood of $x$ in $X$.

Let $R_{i}^{k}(k=i, i+1, \cdots)$ denote the space obtained from $T_{i}$ by applying $f$ to $T_{i}-T_{k}$ and leaving $T_{k}$ alone; i.e., all points of an $\operatorname{arc}$ of $f^{-1}(C) \cap\left(T_{i}-T_{k}\right)$ are identified and only such. In an obvious manner, any continuous sphere $\phi\left(S^{r}\right)$ in $D_{i}-x$ can be considered to be in some $R_{\mathfrak{i}}^{k}-\gamma$. From the definition of the $R_{i}^{k}$, it follows that $f$ induces maps

( $\left.{ }^{3}\right)$ We give an open manifold here, but since it is homeomorphic with $E^{3}$ outside of a bounded set, the 1-point compactification will give a closed manifold.

(4) Smale's theorem states that if $X$ and $Y$ are arcwise connected, locally compact, separable metric spaces, $f: X \rightarrow Y$ is proper and onto, $X$ is $L C^{n}$, and for each $y+Y, f^{-1}(y)$ is $L C^{n-1}$ and $(n-1)$-connected, then $Y$ is $L C^{n}$ and $f$ induces isomorphisms on the homotopy groups up through dimension $n-1$ (and a homomorphism onto for dimension $n$ ). 


$$
\eta_{i}^{r}:\left(R_{i}^{i+r}-\gamma\right) \rightarrow\left(R_{i}^{i+r+1}-\gamma\right) .
$$

Also we have inclusion maps

$$
\tau_{i}^{r}:\left(R_{i}^{i+r}-\gamma\right) \rightarrow\left(R_{i-1}^{i+r}-\gamma\right) .
$$

The injection map $j_{*}^{4}:\left(D_{i+1}-x\right) \rightarrow\left(D_{i}-x\right)$ may be studied through the medium of the $\eta$ and $\tau$ maps, by virtue of the following lemma.

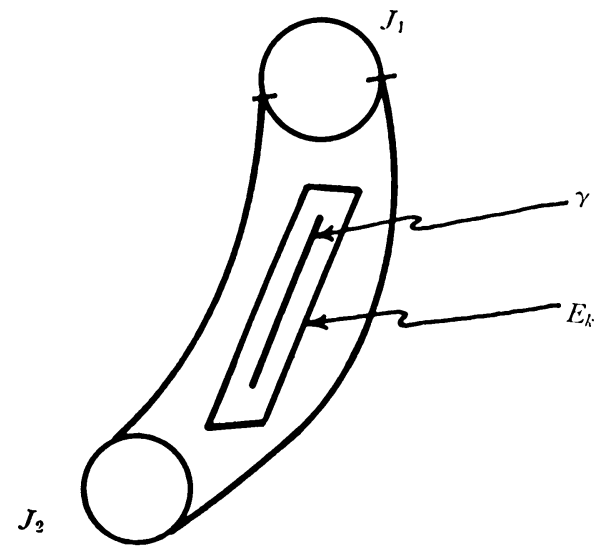

FIGURE 2

Lemma 3.2. (1) $D_{i}-x=\lim _{r}\left(R_{i}^{i+r}-\gamma\right)$,

(2) $j_{*}^{i}=\lim _{r} \tau_{i}^{r_{*}}$ for each $i$.

Proof. The maps in the following diagram commute.

$$
\begin{aligned}
& R_{i}^{i+2}-\gamma \stackrel{\eta_{i}^{2}}{\longrightarrow} R_{i}^{i+3}-\gamma \stackrel{\eta_{i}^{3}}{\longrightarrow} R_{i}^{i+4}-\gamma \longrightarrow \\
& \uparrow \tau_{i+1}^{1} \quad \uparrow \tau_{i+1}^{2} \quad \uparrow \tau_{i+1}^{3} \\
& R_{i+1}^{i+2}-\gamma \stackrel{\eta_{i+1}^{1}}{\longrightarrow} R_{i+1}^{i+3}-\gamma \stackrel{\eta_{i+1}^{2}}{\longrightarrow} R_{i+1}^{i+4}-\gamma \longrightarrow
\end{aligned}
$$

The conclusions of the lemma are automatic.

Lemma 3.3. Each $\eta_{t}^{r}$ is a homotopy equivalence.

Proof. The spaces $R_{i}^{i+r}-\gamma$ are $L C^{\infty}$ by Lemma 3.1. The result now follows from the theorem of Smale used in Lemma 3.1 and a theorem of J. H. C. Whitehead [11] which implies that if a map induces isomorphisms of the homotopy groups, then the spaces have the same homotopy type (for spaces dominated by $\mathrm{CW}$ complexes).

LEMma 3.4. $T_{k}-\boldsymbol{\gamma}$ is deformable in $R_{k-1}^{\mathbf{k}}-\boldsymbol{\gamma}$ onto $E_{k}-\boldsymbol{\gamma}$. 
Proof. See Figure 2.

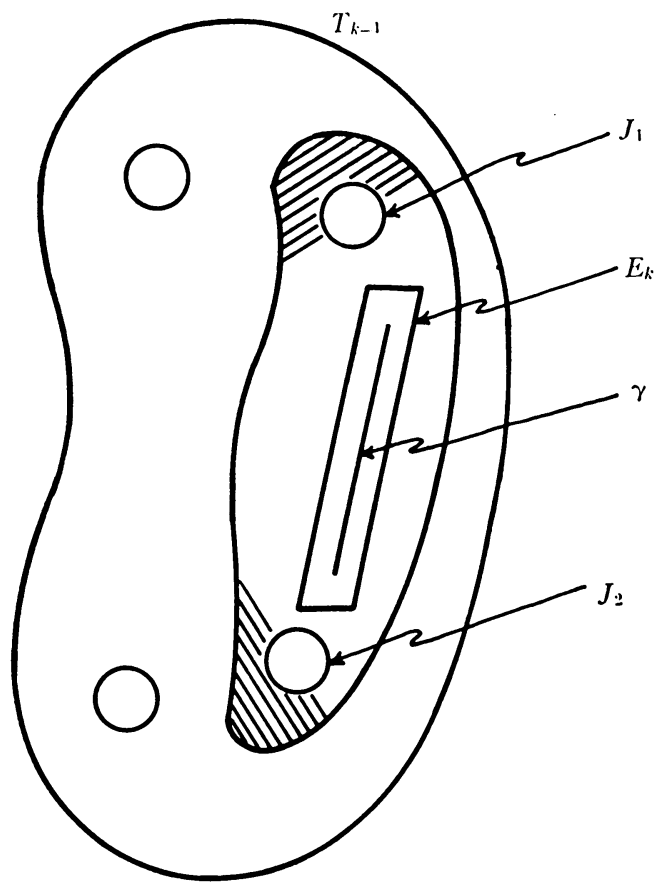

FIGURE 3

$J_{1}$ and $J_{2}$ are simple closed curves on the holes in $T_{k}$ as shown. By a contraction of $T_{k}$ in itself we get a configuration consisting of a 3-cell $E_{k}^{\prime}$ and 2 arcs which lie on $J_{1}$ and $J_{2}$ as shown in Figure 3.

During the contraction $J_{1}$ and $J_{2}$ remain fixed, as do all points of $T_{k}$ except those in the shaded area about $J_{1}$ and $J_{2}$ as shown in Figure 2.

Now $R_{k-1}^{k}$ is obtained from $T_{k-1}$ by shrinking all arcs $f(x)$ not in $T_{k}$ to points. Thus points on the boundary of $T_{k}$ map into themselves, and since the curves $J_{1}$ and $J_{2}$ are deformable on $T_{k-1}-T_{k}$ onto the portion on $E_{k}^{\prime}$, the same is true in $R_{k-1}^{k}-\gamma$. Finally, $E_{k}{ }^{\prime}$ is now deformable in $T_{k}-\gamma$ onto $E_{k}$.

THEOREM. The space $X$ is a 3-dimensional homotopy manifold which is not locally euclidean at all points.

Proof. By definition, $X$ is a 3-dimensional homotopy manifold if (1) $\operatorname{dim} X$ $=3$, (2) $X$ is $L C^{3}$, (3) at each $x \in X$, if $U$ is a neighborhood of $x$ then there exist neighborhoods $V$ and $W$ of $x$ such that $U \supset V \supset W$ and $\pi_{r}(W-x)$ has image isomorphic to $\pi_{r}\left(S^{2}\right)$ in $\pi_{r}(V-x)$ (under the inclusion map).

(1) is clearly satisfied, since $X$ is 3-dimensional at all $x \in X-C$, and for $x \in C$ the neighborhoods of type $T_{i}$ have 2 -dimensional boundaries. No $x \in C$ 
can have arbitrarily small neighborhoods with boundaries of dimension less than 2 because $X$ satisfies Theorem 3.9 of $[12$, p. 215].

(2) was established in Lemma 3.1.

(3) follows from Lemmas 3.3 and 3.4 .

\section{BiBLIOGRAPHY}

1. R. H. Fox and E. Artin, Some wild cells and spheres in three-dimensional space, Ann. of Math. vol. 49 (1948) pp. 979-990.

2. E. G. Begle, The Vietoris mapping theorem for bicompact spaces, Ann. of Math. vol. 51 (1950) pp. 534-543.

3. R. H. Bing, $A$ decomposition of $E^{3}$ into points and tame arcs such that the decomposition space is topologically different from $E^{3}$, Ann. of Math. vol. 65 (1957) pp. 484-500.

4. S. Eilenberg and R. L. Wilder, Uniform local connectedness and contractibility, Amer. J. Math. vol. 64 (1942) pp. 613-622.

5. H. B. Griffiths, Local topological invariants, Proc. London Math. Soc. Ser. 3 vol. 25 (1935) pp. 467-485. 61-89.

6. - A contribution to the theory of manifolds, Michigan Math. J. vol. 2 (1953) pp.

7. V. L. Klee, Some topological properties of convex sets, Trans. Amer. Math. Soc. vol. 78 (1955) pp. 30-45.

8. M. H. A. Newman, Boundaries of ULC sets in euclidean n-space, Proc. Nat. Acad. Sci. vol. 34 (1948) pp. 193-196.

9. S. Smale, $A$ Vietoris mapping theorem for homotopy, Proc. Amer. Math. Soc. vol. 8 (1957) pp. 604-610.

10. J. H. C. Whitehead, Note on a theorem due to Borsuk, Bull. Amer. Math. Soc. vol. 54 (1948) pp. 1125-1132.

11. - Combinatorial homotopy I, Bull. Amer. Math. Soc. vol. 55 (1949) pp. 213-245.

12. R. L. Wilder, Topology of manifolds, Amer. Math. Soc. Colloquium Publications, vol. $32,1949$.

13. - - Montone mappings of manifolds, Pacific J. Math. vol. 7 (1957) pp. 1519-1528.

University of Georgia, Athens, Ga.

UNIVERSITY OF MichigAN, Ann Arbor, Mich. 